\title{
Determinación de la vulnerabilidad sísmica en las edificaciones de la zona urbana del distrito de Jangas, aplicando el sistema de información geográfica
}

Determination of the seismic vulnerability of the buildings in the urban area of the district Jangas, applying geographic information system

Tito Tinoco Meyhuay ${ }^{1}$, Pedro Colonia Cerna ${ }^{1}$ y Pedro Tinoco Gonzales ${ }^{1}$

\section{RESUMEN}

El sismo es uno de los peligros naturales que debido a su magnitud e intensidad causa destrucción y muerte, que a lo largo de la historia y aun en nuestros días no se puede predecir con exactitud cuándo, dónde y de qué magnitud se producirá un futuro evento sísmico. Existen ciertos métodos cualitativos y cuantitativos que permiten reducir los riesgos debido a un evento sísmico, como es la prevención y es responsabilidad de nuestras autoridades locales trabajar en la reducción y/o mitigación de los riesgos. Para la determinación de la Vulnerabilidad Sísmica en las edificaciones de la zona urbana del distrito de Jangas, se aplicó el método del Índice de Vulnerabilidad planteado por los estudiosos italianos Benedetti y Petrini, para lo cual se elaboró la cartografía de la zona urbana en el datum WGS84 y mediante el análisis de once parámetros y la aplicación del SIG para la elaboración de mapas temáticos se ha determinado que, el 21,6\% de las viviendas se encuentra en la clase A, con una vulnerabilidad baja, el 39,0\% en la clase $\mathrm{B}$, con una vulnerabilidad media, el $34,9 \%$ en la clase $\mathrm{C}$, con una vulnerabilidad alta y el $4,5 \%$ en la clase D, con una vulnerabilidad muy alta. Teniendo en cuenta el índice de vulnerabilidad y una aceleración horizontal del terreno de 0,32 gr., se obtuvo que 112 viviendas $(38,4 \%)$ sufrirían el colapso total y 180 viviendas $(61,6 \%)$ seguirían en pie con algún daño estructural. De acuerdo al índice de daño calculado y teniendo en cuenta el precio por metro cuadrado de construcción, si ocurriera un sismo con las características descritas los daños económicos ascenderían a la suma de \$/.54437,440.00.

Palabras clave: sismo; vulnerabilidad; vulnerabilidad sísmica; índice de vulnerabilidad; índice de daños.

\begin{abstract}
The earthquake is one of the natural hazards due to its magnitude and intensity causes destruction and death, that throughout history and even today cannot predict exactly when, where and how large a future seismic event will occur. Certain qualitative and quantitative methods to reduce the risks due to a seismic event, as is prevention and is the responsibility of our local authorities to work in reducing and/or risk mitigation. To determine the Seismic Vulnerability of buildings in the urban area of the district Jangas, the Vulnerability Index method proposed by Italian scholars Benedetti and V. Petrini was applied., For which the mapping of the urban area is developed in the WGS84 datum and by analyzing eleven parameters and application of GIS to prepare thematic maps was determined that $21,6 \%$ of households are in Class A, with low vulnerability, $39,0 \%$ are in class $\mathrm{B}$, mean vulnerability, $34,9 \%$ are in class $\mathrm{C}$, with a high
\end{abstract}

1 Universidad Nacional «Santiago Antúnez de Mayolo». Huaraz, Perú. 
vulnerability and 4,5\% are in class D with very high vulnerability. Given the vulnerability index and a horizontal ground acceleration of 0,32 gr. was obtained that 112 houses $(38,4 \%)$ had a complete collapse and 180 households (61.6\%) follow up with some structural damage. According to the calculated damage index and considering the price per square meter of construction, if an earthquake occurred with the characteristics described economic damages amount to the sum of ${ }^{5 / 54^{\prime}} 437,440.00$.

Keywords: earthquake; vulnerability; seismic vulnerability; vulnerability index; damage index.

\section{ICHIKLLACHAW}

Patsa kuyuyqa allaapa mantsanaypaqmi, allaapa kuyuyninqa tsuqanmi hina wanutsikunmi, unaypitam tsay tsuqayqa kashqa, tsayqa kanankamaran mantsatsikun, alliqllaqa musyantsikraqtsu imay, maychaw, imanaw kay patsapiq ñawpaman patsa kuyuy kanqanta, musyanapaq kanmi kuwalitatibuwan kuwantitatibu mitudu nishqankuna, kay mantsakaykunata uchuktsaatsinapaq patsa kuyuykaptin, imanawmi tsay mana kananpaq pushaqkuna markantsikchaw uchukyaananpaq uryayanman.

Tsay mana alli patsa kuyuy rikaanapaq tsay hatun wayikuna sharitsiychaw kikin Hangas distritunchawmi rurakashqa, kay mana alli kayninta indisi mituduwanmi rurakashqa Benedettiwan V. Petrini runakunapa yachay ashipakuyninwanmi. Tsaypaq kartugrahuwiyata rurakarqan suna urbanachaw WGS84, tsaypaq chunka huk paramitrukunata rurarqan shumaq aplikayaananpaq, tsay SIGpa shumaq maapakunata rurayaananpaq, tsay 21,6\% llapan wayikunapita A laayaman kan, kaypaqa ichikllam mantsaynin kan, 39,0\% B kaq laayachaw tarikan, kaypaqa alliran mantsaynin kan, 34,9\% C kaq laayachaw tarikan, kaypaqa hatunnam mantsaynin kan, 4,5\% D kaq laayachaw tarikan, kaykaqchawqa allaapa atskanam mantsaynin kapun. Tsay llapan mantsaypawan patsa kinraypa hatunyayninta rikaykurninqa tsay llapan kanaq 0,32 gr, tsaypiq 112 wayikunachaw (38,4\%) yarqurqan, llapan hiqayaanaq 180 wayikuna, (61,6\%) mana huchushpa, imallanllam ichik dañukunallawanmi. Llapan dañukuna kanaq chusku mitru kuchuyuq wayi rurashqanchawmi. Kanan tsaynaw yapay huk patsa kuyuy shamuptinqa

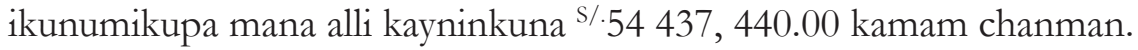

Pushaq shimikuna: patsa kuyuy; tsuqi patsapa kuyuyninpa mantsaynin; aykaman tsay mantsaynin chan; imanaw dañukunapa kanqankuna.

\section{INTRODUCCIÓN}

El distrito de Jangas se encuentra ubicado en la provincia de Huaraz, Región Áncash, en el ámbito del Callejón de Huaylas y, según los antecedentes históricos, corresponde a una zona de alta sismicidad, tal como lo establece el Ministerio de Vivienda, Construcción y Saneamiento (2006), la Norma E-030, Mapa de Zonificación Sísmica (Zona 3) y que en el año de 1970 fue totalmente destruida, con aproximadamente 75,000 víctimas a lo largo del Callejón de Huaylas; por lo que es latente el riesgo de ocurrencia de un evento sísmico igual o superior. Por otro lado, el crecimiento descontrolado y sin planificación de la zona urbana del distrito de Jangas, sin criterios antisísmicos, falta de control en las construcciones nuevas, mantenimiento deficiente en las construcciones existentes hacen que la vulnerabilidad física sea elevada, la mayoría de las viviendas y la infraestructura de servicio social se construyen con nula o baja calidad de diseño 
y materiales locales, sin una adecuada asesoría técnica y limitándose en la mayoría de casos a contratar mano de obra no calificada.

La investigación se realizó en la zona urbana del distrito de Jangas, teniendo como objeto de estudio o análisis las edificaciones existentes para determinar la vulnerabilidad sísmica, empleando el método del Índice de Vulnerabilidad de Benedetti y Petrini, tal como establece Chavarria (2001), los cuales fueron plasmados en mapas temáticos que permitieron la identificación de los diferentes grados de vulnerabilidad, de acuerdo a la Dirección Nacional de Prevención DIAPRE - Unidad de Estudios y Evaluación de Riesgos - UEER (2006), utilizando el sistema de información geográfica. Debido a que el área de estudio se encuentra en una zona eminentemente sísmica y que hasta la actualidad no se ha registrado eventos sísmicos de gran magnitud, el presente trabajo contribuirá a la prevención, teniendo en cuenta los índices de vulnerabilidad sísmica, el cálculo del índice de daños, pérdidas económicas y su posterior procesamiento con la tecnología SIG, luego servirán de instrumentos de gestión al Gobierno Local de Jangas y a la población en general.

\section{MATERIALES Y MÉTODOS}

Investigación descriptiva, no experimental y transversal, cuyas unidades de estudio fueron las viviendas ubicadas en el ámbito de la zona urbana del distrito de Jangas, Provincia de Huaraz, Región Áncash, cuyas coordenadas de referencia de la Plaza de Armas en el datum WGS84 son: E: 216,958.37, N: 8’959,812.01 y una altitud de $2,808.00 \mathrm{msnm}$.

Para la recolección de datos se utilizaron libretas de campo, fichas de vulnerabilidad, imagen satelital de la zona de estudio y el plano catastral preliminar de la zona urbana, los cuales fueron georreferenciados y homogenizados en el datum correspondiente.

El proceso metodológico, adaptado de Castro (2011) y Olarte (2002), consistió en el diagnóstico preliminar del distrito de Jangas, generación de la cartografía de la zona urbana del Distrito de Jangas, aplicación del formulario de vulnerabilidad, cálculo del índice de vulnerabilidad, elaboración de mapas temáticos y el cálculo del índice de daños y pérdidas económicas.

\section{RESULTADOS}

El distrito de Jangas tiene una extensión de $62,33 \mathrm{Km}^{2}$ y perímetro de 38,44 $\mathrm{Km}$. Según el INEI, Censos Nacionales 2007 XI de Población y VI de Vivienda, la población del Distrito de Jangas es de 4,403 habitantes; cuenta con los siguientes servicios básicos: agua potable, desagüe y electricidad. Su clima es propio de la sierra del Perú, con una temperatura anual de $11^{\circ}$ a $22^{\circ} \mathrm{C}$ y una precipitación anual de $550 \mathrm{~mm}$. a 900 $\mathrm{mm}$. En la zona urbana predomina el tipo de suelo GM, con un contenido de grava de $53,23 \%$, arena de $33 \%$ y finos de $13,77 \%$, siendo la presión admisible del terreno de $1,85 \mathrm{Kg} / \mathrm{cm}^{2}$ (capacidad portante alta).

Para la delimitación de la zona de trabajo y el establecimiento de Manzanas: A, B, C, D, E, F y G se tuvo en cuenta el Plano Catastral elaborado por COFOPRI en el año 2004, el levantamiento del plano catastral ejecutado por la Municipalidad Distrital de Jangas el año 2013, la delimitación preliminar de la Zona Urbana de Jangas realizada por el INEI, el levantamiento de datos con GPS e imágenes satelitales de Google Earth. 
Teniendo en cuenta la cartografía de la zona urbana del distrito de Jangas y las fichas de encuesta (formulario de vulnerabilidad), adaptado de Chavarria (2001), se analizaron 292 viviendas y se obtuvo el siguiente resultado:

Tabla 1. Número de viviendas por parámetro y clases

\begin{tabular}{clcccc}
\hline \multirow{2}{*}{$\begin{array}{c}N^{\circ} \\
\text { Parámetro }\end{array}$} & \multicolumn{3}{c}{ Descripción } & \multicolumn{3}{c}{$\mathbf{N}^{\circ}$ Viviendas por Clase } \\
\cline { 3 - 6 } & & $\mathbf{A}$ & $\mathbf{B}$ & $\mathbf{C}$ & $\mathbf{D}$ \\
\hline 1 & Organización del sistema resistente & 41 & 126 & 112 & 13 \\
2 & Calidad del sistema resistente & 42 & 125 & 112 & 13 \\
3 & Resistencia convencional & 63 & 118 & 98 & 13 \\
4 & Posición del edificio y cimentación & 179 & 96 & 16 & 1 \\
5 & Diafragmas horizontales & 140 & 31 & 107 & 14 \\
6 & Configuración en planta & 64 & 78 & 106 & 44 \\
7 & Configuración en elevación & 252 & 27 & 0 & 13 \\
8 & Distancia máxima entre los muros & 79 & 158 & 36 & 19 \\
9 & Tipo de cubierta & 139 & 39 & 92 & 22 \\
10 & Elementos no estructurales & 137 & 21 & 110 & 24 \\
11 & Estado de conservación & 139 & 35 & 105 & 13 \\
\hline
\end{tabular}

Para calcular el índice de vulnerabilidad se elaboró once mapas temáticos que corresponden a cada uno de los parámetros estudiados y asignando rangos para el índice de vulnerabilidad se obtuvo el mapa de índice de vulnerabilidad.

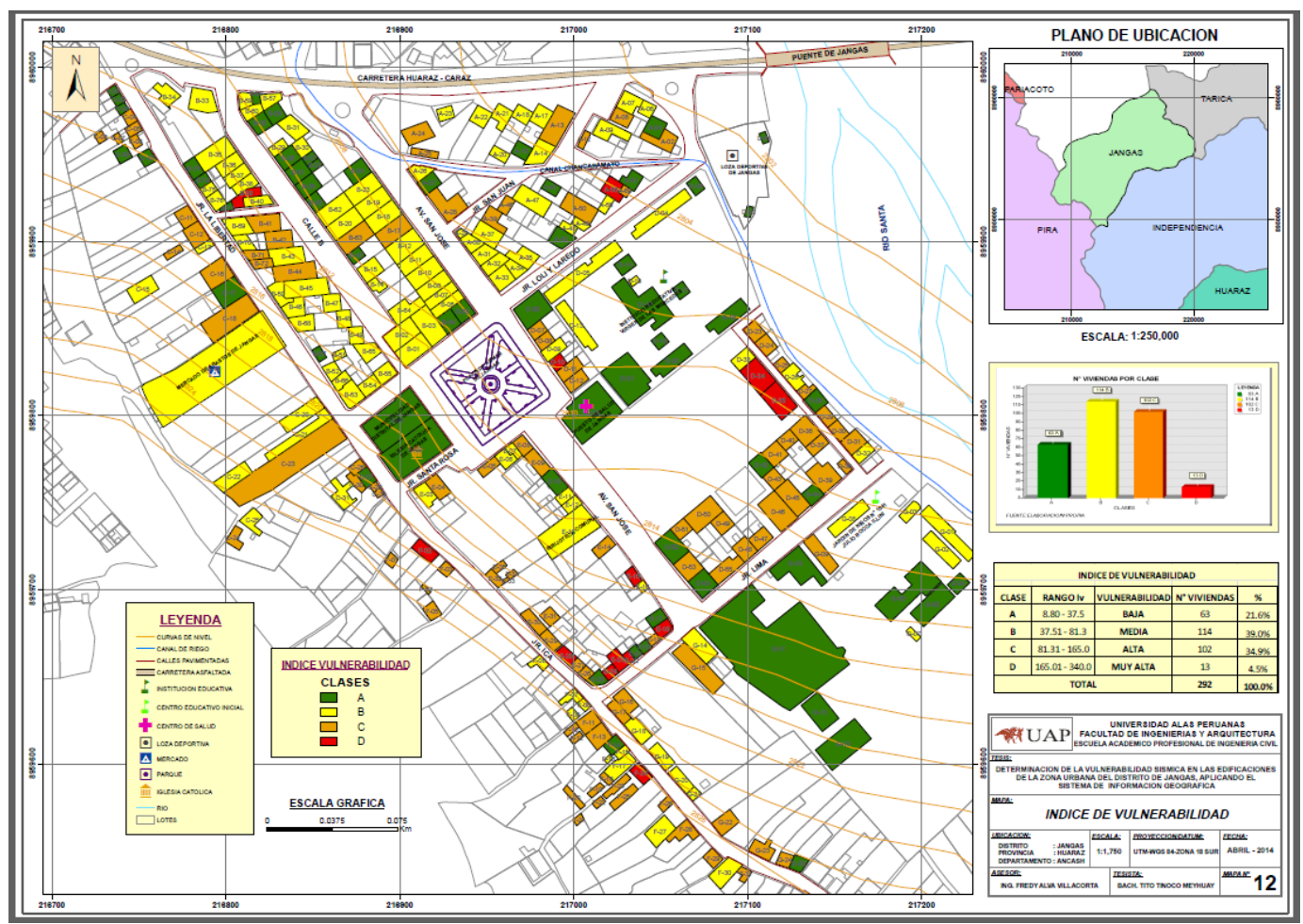

Figura 1. Índice de vulnerabilidad 
Tabla 2. Índice de vulnerabilidad

\begin{tabular}{ccccc}
\hline CLASE & RANGO Iv & VULNERABILIDAD & N $^{\circ}$ VIVIENDAS & \% \\
\hline A & $8,80-37,5$ & BAJA & 63 & $21,6 \%$ \\
B & $37,51-81,3$ & MEDIA & 114 & $39,0 \%$ \\
C & $81,31-165,0$ & ALTA & 102 & $34,9 \%$ \\
D & $165,01-340,0$ & MUY ALTA & 13 & $4,5 \%$ \\
\hline & TOTAL & & $\mathbf{2 9 2}$ & $\mathbf{1 0 0 . 0 \%}$ \\
\hline
\end{tabular}

El daño es expresado en una escala normalizada de $0<d<1$, teniendo como referencia a Chavarria (2001), y representa el costo necesario para recuperar la condición inicial referido al valor de la vivienda. Valores altos de «d» $(0,8-1,0)$ se consideraron equivalentes al colapso total del edificio. Los cálculos se realizaron para aceleraciones horizontales de 0,10 gr., 0,32 gr. y 0,40 gr., los resultados se observan en el siguiente cuadro:

Tabla 3. Índice de vulnerabilidad normalizado

\begin{tabular}{cccc}
\hline Iv Normalizado & Id (a=0,10 $\mathbf{g r})$ & $\mathbf{I d}(\mathbf{a}=\mathbf{0 , 3 2} \mathbf{g r} \mathbf{)}$ & $\mathbf{I d}(\mathbf{a}=\mathbf{0}, \mathbf{4 0} \mathbf{g r .})$ \\
\hline 100 & 0,74 & 2,63 & 3,32 \\
90 & 0,63 & 2,32 & 2,93 \\
80 & 0,54 & 2,03 & 2,57 \\
70 & 0,45 & 1,75 & 2,22 \\
60 & 0,38 & 1,51 & 1,92 \\
50 & 0,31 & 1,30 & 1,66 \\
40 & 0,25 & 1,10 & 1,40 \\
30 & 0,20 & 0,92 & 1,19 \\
20 & 0,16 & 0,77 & 1,00 \\
10 & 0,12 & 0,65 & 0,84 \\
0 & 0,09 & 0,55 & 0,71 \\
\hline
\end{tabular}

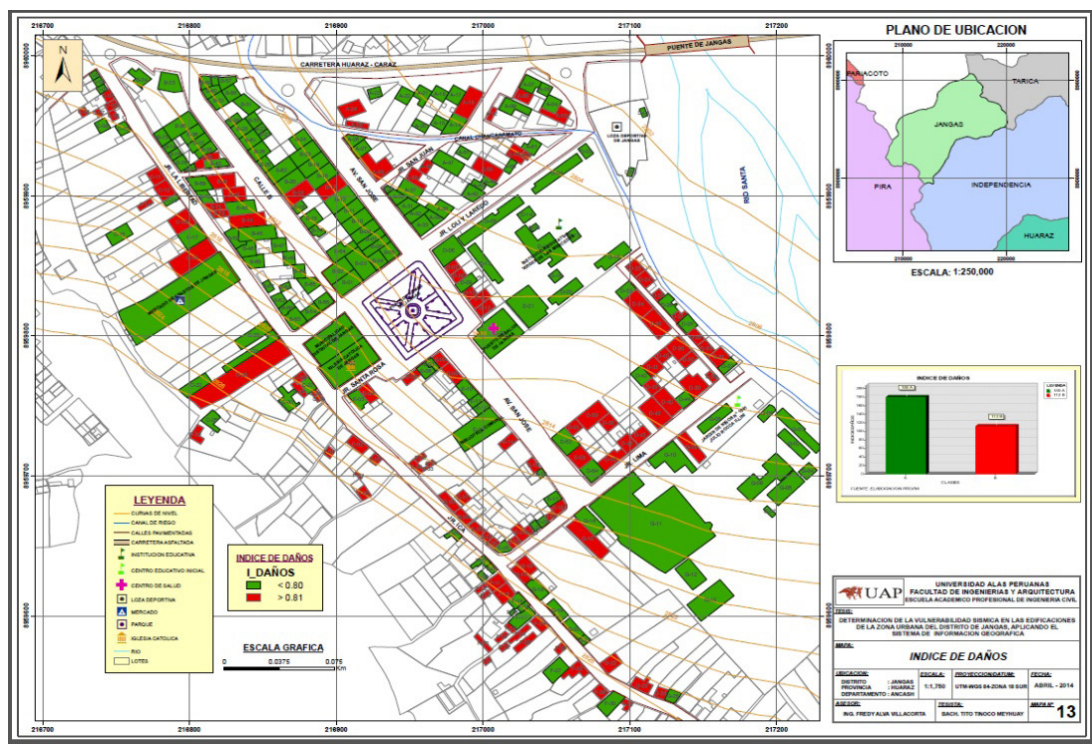

Figura 2. Índice de daños 
Los cálculos de las pérdidas económicas se realizaron para cada vivienda, teniendo en cuenta el daño producido por la aceleración de la horizontal de 0.32 gr. y el costo de construcción de las viviendas de adobe y material noble, siendo para el primer caso

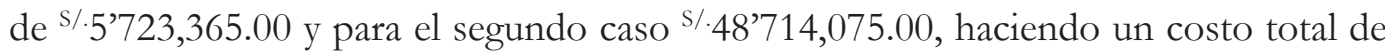
s/.54’437,440.00.

Tabla 4. Pérdidas económicas por manzana

\begin{tabular}{|c|c|c|c|}
\hline MANZANA & ADOBE $\left({ }^{\mathrm{S} /}\right)$ & LADRILLO $\left({ }^{\mathrm{s} /}\right)$ & COSTO PARCIAL $\left({ }^{\mathrm{S} /}\right)$ \\
\hline A & $900,475.00$ & $3^{\prime} 935,225.00$ & $4^{\prime} 835,700.00$ \\
\hline B & $706,660.00$ & $13 ’ 203,350.00$ & $13 ’ 910,010.00$ \\
\hline C & $644,000.00$ & $9 ’ 007,225.00$ & 9'651,225.00 \\
\hline D & $1,793,500.00$ & $6 ’ 884,300.00$ & $8^{\prime} 677,800.00$ \\
\hline $\mathbf{E}$ & $642,395.00$ & 4’296,025.00 & 4’938,420.00 \\
\hline $\mathbf{F}$ & $608,335.00$ & 1’354,250.00 & $1^{\prime} 962,585.00$ \\
\hline G & $428,000.00$ & $10^{\prime} 033,700.00$ & $10,461,700.00$ \\
\hline TOTAL & $5 ’ 723,365.00$ & $48 ’ 714,075.00$ & $54^{\prime} 437,440.00$ \\
\hline
\end{tabular}

\section{DISCUSIÓN}

De acuerdo al índice de vulnerabilidad calculado, el 4,5\% de las viviendas presenta una vulnerabilidad muy alta, debido a que no cumplen con el Reglamento Nacional de Edificaciones, Ministerio de Vivienda, Construcción y Saneamiento (2006), es decir, no presentan vigas y columnas de amarre, son viviendas de adobe con una antigüedad mayor de 30 años, que se encuentran en desuso, pero continúan siendo habitadas. Por otro lado, presentan irregularidades en planta y elevación, ubicadas en terrenos con pendientes muy altas, no presentan planos de construcción y fueron ejecutadas por personas empíricas, sin dirección técnica.

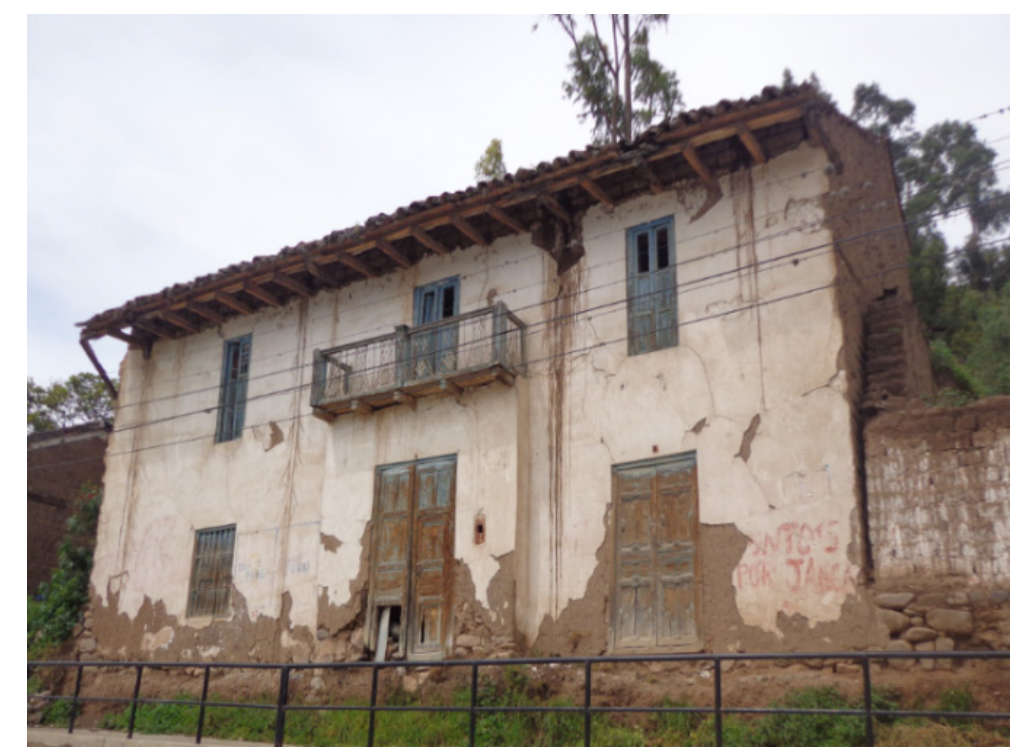

Figura 3. Vivienda de adobe en desuso 
El 34,9\% de las viviendas presenta una vulnerabilidad alta, es decir, algunas características mencionadas en el Reglamento Nacional de Edificaciones. Son viviendas que no presentan vigas y columnas de amarre, que se encuentran distribuidas a lo largo de la zona urbana, son de adobe fabricado en forma artesanal, no son de buena calidad y su construcción se ha realizado sin la presencia de mano de obra calificada, presentan irregularidades en planta y elevación, se ubican en terrenos con pendiente alta, no tienen planos de construcción.

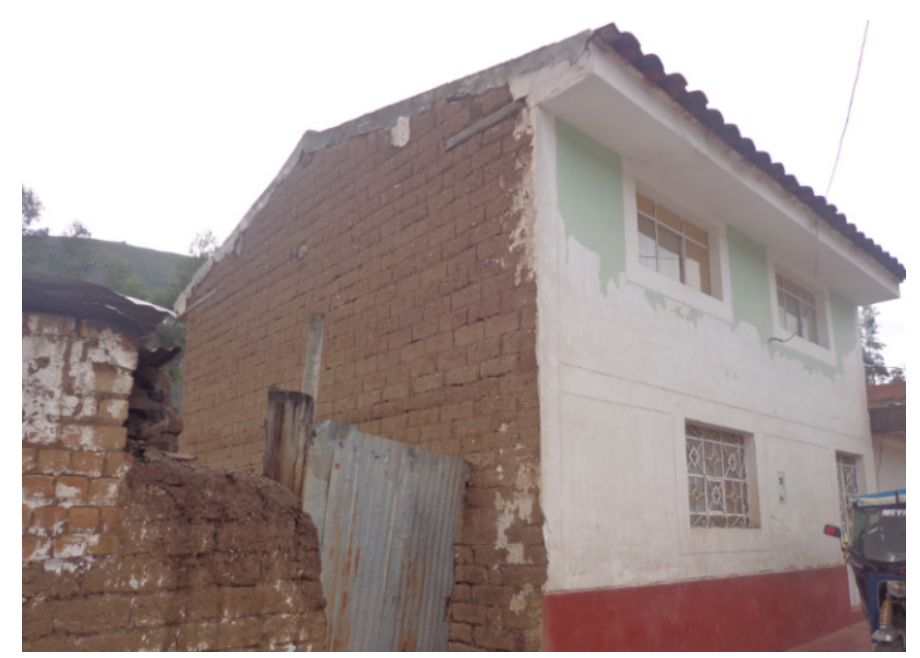

Figura 4. Vivienda que no presenta vigas y columnas de amarre

El 39,0\% de las viviendas se encuentra clasificado en vulnerabilidad media, cumplen con algunas características del Reglamento Nacional de Edificaciones, Ministerio de Vivienda, Construcción y Saneamiento (2006), presentan vigas y columnas de amarre con ciertas deficiencias, son de material noble y han sido construidas sin dirección técnica, con ladrillos artesanales que no cumplen con la resistencia técnica establecida, presentan algunas irregularidades en planta y elevación, con diafragmas horizontales y son viviendas con una antigüedad mayor a 10 años.

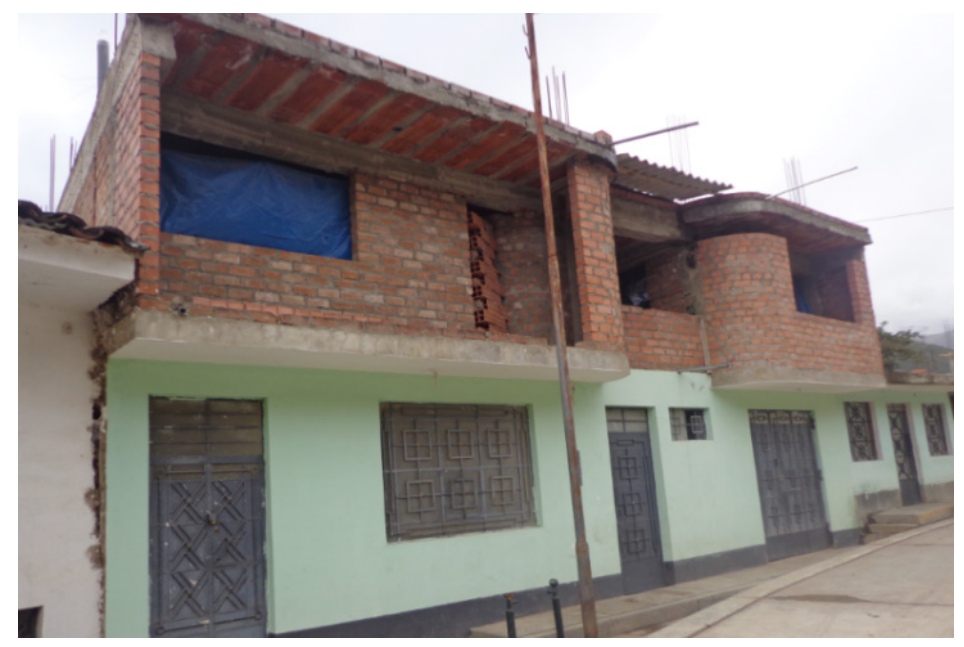

Figura 5. Mampostería con ladrillo artesanal, mortero de espesor no constante 
El 21,6\% de las viviendas presenta vulnerabilidad baja, tienen vigas y columnas de amarre en toda la construcción de la vivienda y son de material noble, con ladrillos procedentes de las fábricas de la ciudad de Lima, cumplen con las normas establecidas por el Reglamento Nacional de Edificaciones, Ministerio de Vivienda, Construcción y Saneamiento (2006), Norma E070 y han sido construidas bajo dirección técnica, presentan planos de construcción, distribución regular en planta y elevación, se encuentran construidas en terrenos con pendiente baja, presentan diafragmas horizontales. En este rango de vulnerabilidad se encuentra la mayoría de las nuevas construcciones de material noble, con una antigüedad de 0 a 5 años y las infraestructuras de servicio público como el local de la Municipalidad, el Mercado de abastos, la Iglesia Católica, el Puesto de Salud, la Institución Educativa Virgen de las Mercedes, entre otras.

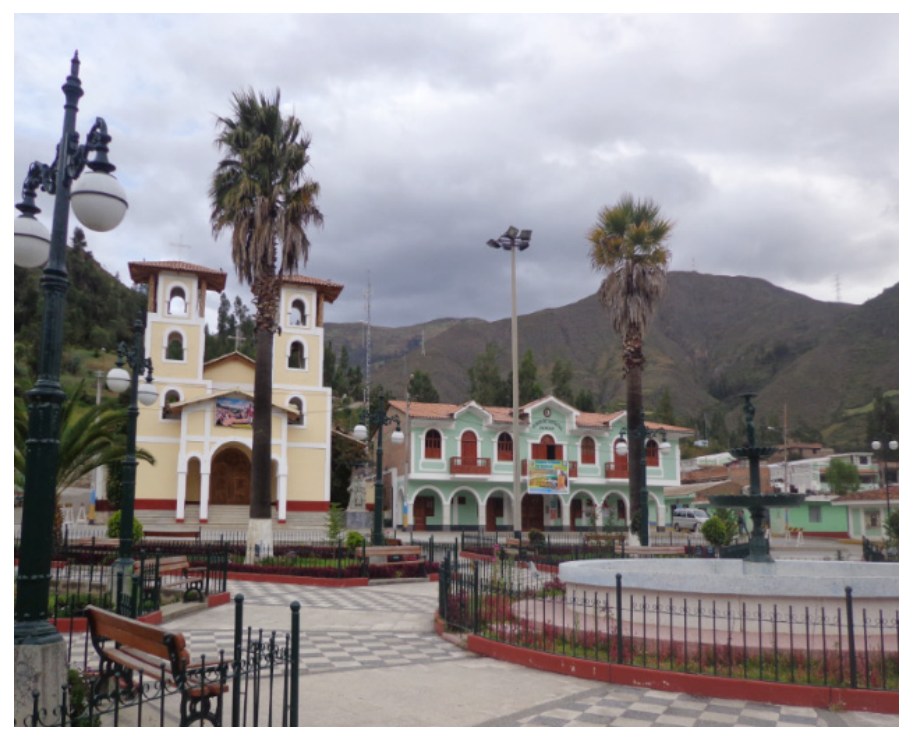

Figura 6. Iglesia de Jangas y Municipalidad Distrital

En base al estudio realizado por Alva y Castillo (1993), para la zona de estudio se tiene el valor de la aceleración horizontal a $=0,32 \mathrm{gr}$; con este valor y tomando en cuenta otras aceleraciones como 0,10 gr. y 0,40 gr. se calcularon los índices de daños.

Teniendo en cuenta que valores mayores a 0,80 del índice de daños y considerando la aceleración horizontal del terreno de 0,10 gr., se realizó una simulación y no se obtuvo colapso de las viviendas de la zona urbana del distrito de Jangas, asegurando que todas las viviendas seguirán en pie y prestando sus funciones. Se asume que los elementos que mayores daños presentarían, serían los elementos no estructurales y la antigüedad de construcción.

Para la aceleración horizontal del terreno de 0,40 gr., que es un sismo de muy alta magnitud y no frecuente, los resultados son muy alarmantes, puesto que todas las viviendas fallarían, trayendo consecuencias desastrosas.

Se ha realizado un análisis minucioso para la aceleración horizontal del terreno de 0,32 gr., cuyo valor es muy similar al evento que ocurrió el 31 de mayo de 1970, encon- 
trándose que 112 viviendas (38,4\%) sufrirían el colapso total y 180 viviendas (61,6\%) seguirían en pie con algún daño estructural. Por otro lado, teniendo en cuenta los costos de construcción por metro cuadrado tanto en adobe como en material noble se ha calculado los daños económicos para las condiciones simuladas, encontrándose que en el caso de las viviendas de adobe los daños económicos cuantificados ascenderían a las suma de ${ }^{\mathrm{S} / 5} \mathbf{5} 723,365.00$ y en el caso de las viviendas de material noble ascenderían a la suma de $\$$ /.48’714,075.00, haciendo un costo total de daños económicos de s/.54’ $437,440.00$.

\section{CONCLUSIONES}

Los materiales que componen estructuralmente las edificaciones de la zona urbana del distrito de Jangas son de dos tipos: materiales de construcción de adobe y de ladrillo; en ambos casos han sido construidas sin la dirección técnica respectiva, a excepción de algunas construcciones de servicio público como: la infraestructura de la Municipalidad, la Posta Médica, la institución educativa Virgen de las Mercedes, entre otras.

Del análisis realizado de los once parámetros y el cálculo del índice de vulnerabilidad que plantea la metodología utilizada en el presente trabajo nos muestra que el 21,6\% de las viviendas se encuentra en la clase A, con una vulnerabilidad baja; el 39,0\% en la clase $\mathrm{B}$, con una vulnerabilidad media, el $34,9 \%$ en la clase $\mathrm{C}$, con una vulnerabilidad alta y el 4,5\% se encuentra en la clase $\mathrm{D}$, con una vulnerabilidad muy alta.

Teniendo en cuenta el índice de vulnerabilidad y una aceleración horizontal del terreno de 0,32 gr., se tiene que 112 viviendas (38,4\%) sufrirían el colapso total y 180 viviendas $(61,6 \%)$ seguirían en pie con algún daño estructural.

De acuerdo al índice de daño calculado y teniendo en cuenta el precio por metro cuadrado de construcción, si ocurriera un sismo con las características descritas, los daños

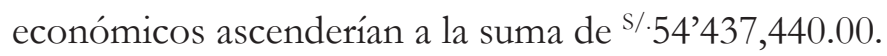

\section{REFERENCIAS BIBLIOGRÁFICAS}

Alva, Jorge y Castillo, Jorge. 2014. Peligro sísmico en el Perú. < http://www.cismid.uni.edu. pe/descargas/redacis/redacis15_a.pdf $>$ [Consulta: 20-02-2014].

Castro, Cristian. 2011. Diagnóstico de la construcción en Ayacucho. Ayacucho: Colegio de Ingenieros del Perú, Consejo Departamental. Capítulo de Ingeniería Civil.

Chavarría, Daniel, y Gómez, Daniel. 2001. Estudio de Vulnerabilidad en Viviendas de 1 y 2 pisos del Barrio Cuarto de Legua en el Cono Cañaveralejo. Tesis Doctoral. Cali: Universidad del Valle de Santiago de Cali.

Dirección Nacional de Prevención DINAPRE. 2006. Manual Básico para la Estimación del Riesgo. Lima: Instituto Nacional de Defensa Civil del Perú (INDECI).

Ministerio de Vivienda, Construcción y Saneamiento. 2006. Reglamento Nacional de Edificaciones. Lima: MVCS. 
||Tito Tinoco, Pedro Colonia y Pedro Tinoco

Olarte, Jorge. 2002. Evaluación de la Vulnerabilidad Sísmica de Áreas Urbano Marginales. Lima: Universidad Nacional de Ingeniería.

Fecha de recepción: 11 de marzo de 2015

Fecha de aceptación: 01 de junio de 2015

\section{Correspondencia}

Tito Tinoco Meyhuay

ttinoco2409@hotmail.com 